PROCEEDINGS OF THE

AMERICAN MATHEMATICAL SOCIETY

Volume 124, Number 12, December 1996, Pages 3739-3743

S 0002-9939(96)03562-9

\title{
HARMONIC MEASURE, INFINITE KERNELS, AND SYMMETRIZATION
}

\author{
JOHN A. VELLING
}

(Communicated by Albert Baernstein II)

\begin{abstract}
The vanishing of area for the infinite Nielsen kernel of an arbitrary open Riemann surface is shown to follow from iteration of a natural geometric operation on the unit disk. This operation compares the distribution of harmonic measure on the boundaries of two related simply connected domains, and is not yet sufficiently well understood.
\end{abstract}

\section{A PROBLEM IN HARMONIC MEASURE}

This paper is an attempt to bring to the attention of the mathematical community a problem which the author has found rather interesting. Consider the following:

Let $\mathbf{D}$ denote the unit disc in the complex plane, and $\mathbf{S}^{1}$ its boundary. We will want to think of $\mathbf{D}$ as furnished with the Poincaré metric, making it a complete metric space of constant curvature -1 . Let $I_{n}, n \in \mathbf{Z}$, be a collection of disjoint $\operatorname{arcs}$ in $\mathbf{S}^{1}$, each with length $<\pi$. Let them be indexed by decreasing length. Each $I_{n}$ is the boundary in $\mathbf{S}^{1}$ of a hyperbolic half-plane, $\mathcal{H}_{n}$, whose other boundary component is a hyperbolic geodesic $J_{n}$ inside $\mathbf{D}$. The indexing may then be thought of as the order of decreasing distance of $J_{n}$ from $0 \in \mathbf{D}$. Since $X=\mathbf{D} \backslash \bigcup_{n} \mathcal{H}_{n}$ is an intersection of hyperbolically convex regions, it is hyperbolically convex and hence simply connected. The condition on length implies that 0 is in the interior of $X$, whence we can map $X$ conformally to $\mathbf{D}$ with $0 \mapsto 0$. Evidently $X$ has rectifiable boundary, so that this Riemann mapping extends to $\partial X$ and $J_{n} \rightarrow I_{n}^{\prime}$, a new arc in $\mathbf{S}^{1}$.

The relationship between the lengths of the $I_{n}$, denoted by $\ell\left(I_{n}\right)$, and the lengths $\ell\left(I_{n}^{\prime}\right)$ is far from clear. A lemma of Loewner implies that $\ell\left(\bigcup_{n} I_{n}\right) \leq \ell\left(\bigcup_{n} I_{n}^{\prime}\right)$, but examples can easily be constructed where some of the $I_{n}$ are shorter than the corresponding $I_{n}^{\prime}$. One may also construct examples where $I_{1}^{\prime}$ is not the longest of the $I_{n}^{\prime}$, so that there is no immediate geometric interpretation of the index on the $I_{n}^{\prime}$. The issue of interest is then the following

Problem. Is $\ell\left(I_{1}\right) \leq \ell\left(I_{1}^{\prime}\right)$ ?

It is believed that the answer to this is 'yes'. We will first present a nice consequence of an affirmative answer, and then several pieces of evidence in support of this conclusion.

Received by the editors May 12, 1995.

1991 Mathematics Subject Classification. Primary 30C85.

Partially supported by NSF grant \# 4401728 and PSC-CUNY grant \#6-64131. 


\section{Several COnsequences of An AFFirmative Answer}

Let $\mathcal{R}_{0}$ be a finite type Riemann surface with boundary, and $\mathcal{R}_{-\infty}$ denote its infinite Nielsen kernel (the meaning of which will be reviewed below). The following is a theorem of J. Cao [2]:

Theorem ([2]). If $\mathcal{R}_{0}$ is a finite type Riemann surface with boundary, $\mathcal{R}_{-\infty}$ has no interior.

Based on the positive resolution of the above problem, we may offer an extension of the above theorem.

Theorem 1. For any Riemann surface $\mathcal{R}_{0}$ of the second kind there is no interior to $\mathcal{R}_{-\infty}$.

Our proof, by much the same reasoning as Cao's but with emphasis on Schwarz's lemma, shows that the topology of $\mathcal{R}_{0}$ is inessential.

We also obtain the following stronger version of Theorem 1 , which we distinguish because of the brevity of the respective proofs.

Theorem 2. For any Riemann surface $\mathcal{R}_{0}$ of the second kind, $\mathcal{R}_{-\infty}$ has no area.

Before demonstrating the above results, we explain several terms.

\section{Riemann surfaces of the Second Kind And Nielsen Kernels}

A Riemann surface of the second kind is conformally a quotient of $\mathbf{D}=\{z \in \mathbf{C}$ : $|z|<1\}$ by a group of orientation preserving Möbius transformations preserving $\mathbf{D}$ and acting discontinuously on $\mathbf{S}^{1}=\partial \mathbf{D}$. We will give such surfaces hyperbolic geometry inherited from $\mathbf{D}$ as a model of $\mathbf{H}^{2}$. The groups acting here are said to be of the second kind, and the domain of discontinuity in $\mathbf{S}^{1}$ for such a group is a union of disjoint arcs. Examples of such surfaces include infinite volume hyperbolic surfaces of finite type.

Henceforth let $\mathcal{R}_{0}=\mathbf{D} / \Gamma_{0}$ be a Riemann surface of the second kind and assume for the moment that the group is non-elementary. The Nielsen kernel of such a Riemann surface, denoted by $\mathcal{R}_{-1}$, is a new Riemann surface of the same topological type as $\mathcal{R}_{0}$. It is constructed as follows: Let $\left\{I_{k}\right\}$ denote the collection of arcs in the domain of discontinuity for the action of $\Gamma_{0}$ on $\mathbf{S}^{1}$. Denote by $J_{k}$ the hyperbolic geodesic spanning the corresponding $I_{k}$. On deleting from $\mathbf{D}$ the hyperbolic halfplanes bounded by the $I_{k}$ and $J_{k}$ we are left with a connected, simply connected, $\Gamma_{0}$-invariant domain in $\mathbf{D}$. The quotient of this domain by $\Gamma_{0}$ is conformally the desired surface $\mathcal{R}_{-1}$. The Nielsen kernel of $\mathcal{R}_{0}$ is empty when $\Gamma_{0}$ is elementary. For more background on this, see any of Halpern's papers concerning this: [7], for example. We will henceforth assume $\Gamma_{0}$ is non-elementary.

One may iterate this procedure to obtain a sequence of Riemann surfaces $\mathcal{R}_{0}$, $\mathcal{R}_{-1}, \mathcal{R}_{-2}, \ldots$ with $\mathcal{R}_{n-1}$ the Nielsen kernel of $\mathcal{R}_{n}(n \leq 0)$. There are thus natural injections $\iota_{n m}: \mathcal{R}_{n} \rightarrow \mathcal{R}_{m}$ obtained from the above procedure, so that $\partial\left(\iota_{(n-1) n}\left(\mathcal{R}_{n-1}\right)\right) \subset \mathcal{R}_{n}$ is a collection of simple geodesics (with respect to the hyperbolic geometry of $\mathcal{R}_{n}$ ) homotopic to ideal boundary components of $\mathcal{R}_{n}$. Evidently $\iota_{n m} \circ \iota_{m l}=\iota_{n l}$. Identifing $\mathcal{R}_{n}$ with all of its images $\iota_{n m}\left(\mathcal{R}_{n}\right)$, we see that $\left\{\mathcal{R}_{k}\right\}_{-\infty}^{n-1}$ is a nested sequence of open sets in $\mathcal{R}_{n}$. The intersection of these will be called $\mathcal{R}_{-\infty}$, the infinite Nielsen kernel. As a topological space with an induced conformal structure (even though $\mathcal{R}_{-\infty}$ may not be a Riemann surface) from the embeddings into the $\mathcal{R}_{n}, \mathcal{R}_{-\infty}$ is independent of the $\mathcal{R}_{n}$ in which it is embedded. 


\section{Proofs of the 'Theorems'}

This development assumes an affirmative answer to the problem. We will also make use of the following versions of Schwarz's lemma:

Lemma 1. Let $A, B$ be subdomains of $\mathbf{D}$ such that $0 \in B \subset A$. If $f: \mathbf{D} \stackrel{1-1}{\longrightarrow} A \subset \mathbf{D}$ and $g: A \rightarrow B$ with $f(0)=g(0)=0$, then $\left|f^{\prime}(0)\right| \geq\left|(g \circ f)^{\prime}(0)\right|$.

Lemma 2. Let $f: \mathbf{D} \stackrel{1-1}{\longrightarrow} \mathbf{D}$ with $f(0)=0$ and $\mathbf{D}_{r}=\{r z:|z| \in \mathbf{D}\} \subset f(\mathbf{D})$ for some $r<1$. Then $\left|f^{\prime}(0)\right|>r$.

Proof of 'Theorem' 1 . Let $\mathcal{R}_{0}$ be any non-elementary Riemann surface of the second kind. Let $p \in \mathcal{R}_{n} \subset \mathcal{R}_{m} \subset \mathcal{R}_{0}$ for fixed $n$. Let $\tilde{\iota}_{n m}: \mathbf{D} \rightarrow \mathbf{D}$ be the lifts of $\iota_{n m}: \mathcal{R}_{n} \rightarrow \mathcal{R}_{m}$ such that 0 is always a lift of $p$. By the problem and Lemma 1 , there exists a constant $c<1$ such that $\left|\tilde{\iota}_{n(n-1)}^{\prime}(0)\right|<c$. (One may take as $A$ in Lemma 1 the domain $\mathbf{D} \backslash$ (the largest half-plane, as viewed from $p \in \mathcal{R}_{0}$, deleted on the creation of $\left.\mathcal{R}_{-1}\right)$. Thus $\left|\tilde{\iota}_{n 1}^{\prime}(0)\right|<c^{n}$. The theorem now follows by the contrapositive of Lemma 2.

To prove 'Theorem' 2 we will need

Lemma 3. Let $\mathbf{D}(n)$ be a family of disks indexed by the nonnegative integers, and $f_{n}: \mathbf{D}(n) \rightarrow \mathbf{D}(n-1)$ be univalent injections with $f_{n}(0)=0$. Assume there is an $r<1$ such that $f_{n}(\mathbf{D}(n))$ always misses a piece of definite area in $\mathbf{D}_{r} \subset \mathbf{D}(n-1)$. With $g_{n}=f_{1} \circ f_{2} \circ \cdots \circ f_{n}, 0$ is not a point of Lebesgue density for $\bigcap_{1}^{\infty} g_{n}(\mathbf{D}(n))$ $\subset \mathbf{D}(0)$.

Proof of Lemma 3. Our hypothesis of missing area in $\mathbf{D}_{r}$ at each step implies, by the Koebe $\frac{1}{4}$-theorem, that there is some $c<1$ so that $f_{n}^{\prime}(0)<c$ for all $n$. We suppose that there is an $\epsilon>0$ such that, for every $n>0, X_{n-1}=\mathbf{D}_{r} \backslash f_{n}(\mathbf{D}(n)) \subset$ $\mathbf{D}(n-1)$ has area $>\epsilon\left(\pi r^{2}\right)$. Then the Koebe distortion theorem tells us that

$$
\frac{\int_{X_{i}}\left|g_{i}^{\prime}\right|^{2} d x d y}{\int_{B_{r}(0)}\left|g_{i}^{\prime}\right|^{2} d x d y} \geq \frac{(1-r)^{8}}{(1+r)^{8}} \cdot \frac{\int_{X_{i}} 1 d x d y}{\int_{B_{r}(0)} 1 d x d y} \geq \frac{(1-r)^{8}}{(1+r)^{8}} \epsilon
$$

i.e., inside of arbitrarily small balls about $0\left(\right.$ since $\left|g_{n}^{\prime}(0)\right|<c^{n} \rightarrow 0$ ) a definite proportion of the area is missing.

Proof of 'Theorem' 2. We apply Lemma 3 at each point of $\mathcal{R}_{-\infty} \subset \cdots \subset \mathcal{R}_{-2} \subset$ $\mathcal{R}_{-1} \subset \mathcal{R}_{0}$ by lifting to the universal covers of the $\mathcal{R}_{n}\left(n \in \mathbf{Z}_{-}\right)$to see that no point of $\mathcal{R}_{-\infty}$ is a point of Lebesgue density. The problem furnishes the area deficiency needed in Lemma 3.

It appears that one can continue in this vein to obtain bounds on the local Hausdorff dimension of $\mathcal{R}_{-\infty}$, since not only are we excising a substantial piece of area at every level but these pieces are very regular. One would thus be able to bound the Hausdorff dimension of the infinite Nielsen kernel for finite type surfaces strictly away from 2 . 


\section{A COMment on the infinite Nielsen extension}

One further consequence of a positive resolution of our conjecture is the ability to state conclusively that the infinite Nielsen extension (reviewed below) cannot arise from certain naive constructions.

The operation of taking Nielsen kernels has an inverse. Thus if we have a Riemann surface, $\mathcal{R}_{0}$, of the second kind, we may find its Nielsen extension, $\mathcal{R}_{1}$, into which $\mathcal{R}_{0}$ naturally injects as Nielsen kernel. For a full description of this process, see [4], [5], or [6], for example. There are again special cases when $\mathcal{R}_{0}=\mathbf{D} / \Gamma_{0}$ for $\Gamma_{0}$ elementary, so we still assume that $\Gamma_{0}$ is non-elementary.

With this assumption, $\mathcal{R}_{1}$ is a surface of the second kind, of the same topological type as $\mathcal{R}_{0}$, and we may iterate the extension operation. We obtain a sequence of surfaces $\mathcal{R}_{1}, \mathcal{R}_{2}, \ldots$ where we may consider $\mathcal{R}_{n} \subset \mathcal{R}_{n+1}$. Thus we also have the limiting surface $\mathcal{R}_{+\infty}=\bigcup_{n \in \mathbf{Z}} \mathcal{R}_{n}$. This is called the infinite Nielsen extension of $\mathcal{R}_{0}$. Bers [1] showed that when $\mathcal{R}_{0}$ is of finite type, i.e. of finite genus with a finite number of boundary curves and punctures, $\mathcal{R}_{+\infty}$ is a surface of the same topological type as $\mathcal{R}_{0}$ but it is quasiconformally distinct - it has punctures where $\mathcal{R}_{0}$ has boundary curves. In particular, it is still hyperbolic. The Nielsen extension map, with $\mathcal{R}_{0} \mapsto \mathcal{R}_{+\infty}$, is surjective to the space of punctured surfaces of the appropriate topological type [6]. We will further restrict ourselves to this setting where $\mathcal{R}_{0}$ is of finite type.

It is reasonable to ask if either the infinite Nielsen extension, with $\mathcal{R}_{0} \mapsto \mathcal{R}_{+\infty}$, or the inverse, which identifies the $\mathcal{R}_{0}$ giving rise to a specific punctured surface as $\mathcal{R}_{+\infty}$, occur in a more naive geometric way. Two possibilities for such in this latter case are:

1. Horocyclic excision: With $\mathcal{R}_{+\infty}=\mathbf{D} / \Gamma_{+\infty}$, every puncture on $\mathcal{R}_{+\infty}$ corresponds to an equivalence class of parabolic fixed points for the action of $\Gamma_{+\infty}$. We may obtain surfaces with boundary curves where $\mathcal{R}_{+\infty}$ has punctures by cutting along horocycles about the punctures.

2. Disk excision: In [3], Haas showed that for a finite genus Riemann surface with boundary curves, $\mathcal{R}_{0}$, there is a unique extension to a surface of the same genus such that the boundary curves of $\mathcal{R}_{0}$ are boundaries of geometric disks. (Geometry is spherical, planar, or hyperbolic depending on genus.) We obtain a punctured surface by removing the centers of these disks, and may reverse this process by excising geometric disks about the centers.

These operations are clearly conformally natural. We ask if the $\mathcal{R}_{0}$ giving rise to $\mathcal{R}_{+\infty}$ through the infinite Nielsen extension may be obtained from $\mathcal{R}_{+\infty}$ by either of these excisions. In both cases, as a corollary of Theorem 1, the answer is seen to be 'no'. This is because the complement of a locally finite collection of disjoint disks in any of $\mathbf{D}, \mathbf{C}$, or $\hat{\mathbf{C}}$ necessarily has interior. Thus we have

Corollary. The family of $\mathcal{R}_{0}$ yielding a fixed $\mathcal{R}_{+\infty}$ by the infinite Nielsen extension is obtained neither by the horocyclic extension nor by the hyperbolic disk extension.

\section{Some supporting EVIDEnce}

To obtain an affirmative answer to the problem, it would suffice, by subordination and a normal families argument, to establish the following conjecture:

Conjecture. Let $I_{1}, I_{2}, \ldots, I_{n}$ be a finite collection of disjoint arcs filling out $\mathbf{S}^{1}$, each of length $<\pi$, and ordered by decreasing length. Let $I_{k}^{\prime}$ be as described in $\S 1$. 
Then $\ell\left(I_{1}\right) \leq \ell\left(I_{1}^{\prime}\right)$, with strict inequality unless the $I_{k}$ are all of the same length and fill out $\mathbf{S}_{1}$ (i.e. the full symmetric case).

We can see that this is true for Möbius images of the full symmetric case as follows.

First note that in the full symmetric case, we may assume that the endpoints of our arcs are at the $n$-th roots of $1, \rho_{n}^{k}=e^{\frac{2 \pi k i}{n}}$. Let $I_{k}$ be the $\operatorname{arc}$ in $\mathbf{S}^{1}$ from $\rho_{n}^{k-1}$ to $\rho_{n}^{k}, J_{k}$ the hyperbolic geodesic between these two points, and $H_{k}$ the hyperbolic half-plane bounded between $I_{k}$ and $J_{k}$. There is a Riemann map from $\mathbf{D} \backslash \bigcup_{1}^{n} H_{k}$ to $\mathbf{D}$ preserving 0 and the $\rho_{n}^{k}$, mapping the radius from 0 to $\rho_{n}^{k}$ (call this $R_{k}$ ) bijectively to itself. Let $f$ denote this map. Let $p \in \mathbf{D}$ be a point in the hyperbolic triangle bounded by $R_{0}, R_{1}$, and $J_{1}$. Now let $h_{1}$ be the harmonic function on $\mathbf{D}$ which is 1 on $I_{1}$ and 0 on the rest of $\mathbf{S}^{1}$, so that $h_{2}=h_{1} \circ f$ is harmonic on $\mathbf{D} \backslash \bigcup_{1}^{n} H_{k}$. In this setting, our problem is equivalent to showing that $h_{1}(p) \leq h_{2}(p)$, with equality holding only when $p=0$.

This is now straightforward by subordination. Since $h_{1}<0$ in $\mathbf{D}$, we have $h_{1}<$ $h_{2}$ on $J_{1}$. Also, since $h_{1}$ is increasing radially on $R_{0}$ and $R_{1}$, and $f$ is an expansion with respect to the hyperbolic metric (Schwarz-Pick lemma), $h_{1} \leq h_{1} \circ f=h_{2}$ on $R_{0}$ and $R_{1}$, with equality only at the origin. Subordination now gives the result in this special case.

One may also show that the above conjecture is true for any four disjoint arcs filling $\mathbf{S}^{1}$, using elliptic functions. A general proof, however, eludes this author.

\section{REFERENCES}

1. Bers, L., Nielsen extensions of Riemann surfaces, Ann. Acad. Sci. Fenn. 2 (1976), 17-22. MR 58:6219

2. Cao, J., The Bers-Nielsen kernels and souls of open surfaces with negative curvature, Michigan Math. J. 41 (1994), 13-30. MR 95d:30085

3. Haas, A., Linearization and mappings onto pseudocircle domains, Trans. AMS 282 (1984), 415-429. MR 86b:30069

4. Halpern, N., On the infinite Nielsen extension of a torus, Comp. Var. 4 (1984), 111-117. MR 86b:30068

5. __ Some remarks on Nielsen extensions of Riemann surfaces, Mich. Math. J. 30 (1983), 65-68. MR 84j:30079

6. A theorem on infinite Nielsen extensions, Proc. AMS 97 (1986), 459-462. MR 88h:32017

7. _ Two theorems on Nielsen cores, Comp. Var. 8 (1987), 123-127. MR 88k:30053

Department of Mathematics, Brooklyn College (CUNy), Brooklyn, New York 11210

E-mail address: jvelling@brooklyn.cuny.edu 